ISSN: $2316-4093$

\title{
Produção de biodiesel de óleo cru refinado e óleo de fritura de soja
}

\author{
Anderson Rosa ${ }^{1 *}$, Amauri Ghellere Garcia Miranda ${ }^{1}$, Cristiano Fernando Lewandoski ${ }^{1}$, \\ Reginaldo Ferreira Santos ${ }^{1}$
}

\footnotetext{
${ }^{1}$ Universidade Estadual do Oeste do Paraná - UNIOESTE, PPGEA - Programa de Pós-Graduação em Energia na Agricultura - Nível Mestrado e Doutorado, Cascavel-PR.
}

*Autor correspondente: andersonrosacb@hotmail.com Artigo enviado em 17/09/2019, aceito em 01/05/2020

\begin{abstract}
Resumo: A busca pela redução de combustíveis que agridam menos o meio ambiente, o biodiesel vem se mostrando uma opção viável financeiramente e sustentável. 0 objetivo deste trabalho foi avaliar o processo de transesterificação de óleo de soja cru e de fritura para a produção de biodiesel, além de avaliar a adequação com as normas nacionais recomendadas para biodiesel. 0 processo de produção do biodiesel adotado foi o de transesterificação utilizando metanol e hidróxido de potássio. Foram dois tratamentos utilizando óleo de soja cru refinado e óleo de soja de fritura com dez fabricações de biodiesel para cada tipo de óleo. Os dados foram submetidos à análise de variância (ANOVA) para as análises químicas e rendimento de biodiesel com significativa a $5 \%$ de probabilidade pelo teste Tukey. Resultados avaliados mostraram que o biodiesel produzido de ambas as fontes avaliadas está de acordo com normas regulamentadoras dispostas pela ANP. Tornando assim estes produtos tecnicamente viáveis para comercialização. 0 rendimento na produção do biodiesel oriundo de óleo refinado cru de soja foi 4,5\% superior ao do óleo residual do processo de fritura de alimento, 93,80\% e $89,60 \%$ respectivamente. Porem os dois tipos de óleo avaliados mostraram-se com uma ótima conversão em biodiesel.
\end{abstract}

Palavras-chave: descarbonificação; fontes energéticas, transesterificação.

\section{Biodiesel production of refined crude and frying soybean oil}

Abstract: The search for the reduction of fuels that harm the environment less, biodiesel has proved to be a financially viable and sustainable option. This article aimed to evaluate the process of transesterification of crude soybean oil and frying for the production of biodiesel, in addition to assessing the adequacy with the national standards recommended for biodiesel. The biodiesel production process adopted was transesterification using methanol and potassium hydroxide. Were two treatments using refined raw soybean oil and frying soybean oil with ten biodiesel fabrications for each type of oil. The data were subjected to analysis of variance (ANOVA) for chemical analyzes and biodiesel yield with a significant $5 \%$ probability by the Tukey test. Evaluated results showed that the biodiesel produced from both evaluated sources is in accordance with regulatory standards set by the ANP. Thus making these products technically viable for commercialization. The yield in the production of biodiesel from crude refined soybean oil was $4.5 \%$ higher than that of residual oil from the food frying process, $93.80 \%$ and $89.60 \%$ respectively. However, the two types of oil evaluated showed an excellent conversion to biodiesel.

Key words: descarbonification, energy sources, transesterification. 


\section{Introdução}

A busca por alternativas menos agressivas ao meio ambiente estão sendo amplamente debatidas e trazendo à tona questões referente a importância do desenvolvimento sustentável. 0 conceito conhecido como Triple Botton Line vem ganhando cenário, onde defende os três pontos chaves para o desenvolvimento sustentável, sendo: aspecto social, ambiental e econômico (Oliveira et al., 2012)

Segundo relatório anual da Agência Nacional de Petróleo, Gás Natural e Biocombustíveis, o biodiesel é uma fonte energética renovável proveniente do processo de transesterificação de óleos orgânicos. Desde 2008 tem sua presença obrigatória no diesel comercializado em nosso país. Onde, atualmente o teor de biodiesel no diesel é de 10\%. No ano de 2018 foram comercializados 5.391 bilhões de litros de biodiesel, volume $25,3 \%$ acima ao ano anterior (ANP, 2019).

O Brasil possui uma quantidade expressiva de espécies de plantas cultivadas que podem servir como matéria prima para produção de biodiesel, estas plantas são também conhecidas como Culturas Energéticas (Santos, 2018). Os combustíveis fósseis são potenciais poluidores ambientais, pois; durante a sua combustão ocorre à liberação de gases de efeito estufa e, na ocasião do derramamento acidental destes combustíveis, ocasiona contaminação da água, do solo e provoca morte de animais (Silva e Freitas, 2008).

0 uso do biodiesel reduz a emissão de poluentes para a atmosfera, assim como a transformação de óleo residual de frituras em combustível, e minimiza o efeito deste sobre o solo e cursos d'água (Lopes et al., 2008). 0 biodiesel resulta na redução significativa da emissão de gases do efeito estufa quando se compara a queima deste combustível com o diesel convencional com uma redução de $36 \%$ da opacidade da fumaça (Lima et al., 2012).

O biodiesel é visto como possível solução para as incertezas quanto ao futuro energético e sua segurança, principalmente aos países em desenvolvimento como o Brasil. Devido à grande semelhança ao diesel, em termos de estrutura química e conteúdo de energia, o biodiesel é compatível com os motores de combustão interna em operação atualmente. Ponto que cria grande vantagem a esta fonte, uma vez que não necessita de modificações para o seu uso (Lam et al., 2009).

Nos processos industriais para produção do biodiesel se destacam a transesterificação de óleo vegetal e a esterificação de um ácido graxo com um álcool de baixo peso molecular, como etanol ou metanol, através de uma reação com a presença ou ausência de um catalisador homogêneo ou heterogêneo (Marulanda-Buitrago e MarulandaCardona, 2015).

0 Brasil encontra-se numa situação privilegiada, pois possui grande extensão territorial e condições climáticas favoráveis ao cultivo de plantas oleaginosas, tornando-se um dos países com potencial para liderar a produção mundial de biodiesel. 0 Brasil é um dos maiores produtores de soja, os principais produtos derivados da soja são o óleo e o farelo, o farelo é amplamente utilizado na alimentação animal, e o óleo de soja é um dos óleos mais utilizados no preparo de alimentos tanto nas industrias como domiciliar pelo consumidor final (Souza et al., 2019).

A possibilidade de um maior aproveitamento do resíduo gerado pelo óleo de soja na indústria do biodiesel é ambientalmente interessante, apresentando-se como um outro mercado consumidor deste insumo, 
evitando assim que ocorra um descarte incorreto deste material, o óleo de cozinha usado e o resíduo de óleo de soja usado pela indústria alimentícia se for descartado em locais inadequados pode acarretar no entupimento das redes de esgoto, prejudicar o tratamento de esgoto, contaminar o solo e a água de rios e lagos, e ainda ocasionar a morte da fauna e da flora aquática.

Assim, a possibilidade de reaproveitamento óleos residuais de fritura para obtenção de biodiesel é muito vantajoso, pois transforma resíduo descartável em fonte de en ergia, devido a esse cenário o objetivo deste trabalho foi avaliar o processo de transesterificação de óleo de soja cru e de fritura para a produção de biodiesel, além de avaliar a adequação com as normas nacionais recomendadas para biodiesel.

\section{Material e métodos}

0 experimento foi conduzido no mês de abril de 2019 no Núcleo de Inovações Tecnológicas - NIT do Programa de Pós-graduação em Engenharia de Energia na Agricultura PPGEA da Universidade Estadual do Oeste do Paraná, localizado em Cascavel$\mathrm{PR}$, foram dois tratamentos utilizando óleo de soja cru refinado e óleo de soja de fritura com dez fabricações de biodiesel para cada tipo de óleo.

A reação utilizada para a fabricação do biodiesel foi transesterificação, utilizando metanol e hidróxido de potássio (KOH) como catalisador. Os dois óleos foram aquecidos e quando chegaram a temperatura de aproximadamente $80^{\circ} \mathrm{C}$, foi adicionado o catalisador e deixado reagir sob agitação por 10 minutos.

Após os 10 minutos, com o processo de transesterificação completo, o biodiesel foi colocado em funil de decantação por um período de 24 horas, para a separação da glicerina. Uma vez finalizado o processo de decantação, a glicerina foi retirada do biodiesel, por meio de gravimetria, abrindo a torneira do funil de decantação.

Concluído este processo, o biodiesel foi colocado em garrafas, separadas, e seguiram para as análises laboratoriais. Nesta etapa, foi feita a medição do volume de rendimento do biodiesel com uma proveta graduada, sendo este a relação volumétrica do óleo utilizado para produção do biodiesel e o volume real de biodiesel produzido. Análise de densidade foi realizada por gravimetria, com o auxílio de uma balança e uma proveta graduada. A massa específica foi realizada com o auxílio de um densímetro usado para medição de diesel comum.

$\frac{\text { mass } a}{\text { volume }}=$ densidade $\left(\frac{g}{m l}\right)$

A determinação de acidez foi realizada pelo método de titulação, onde foram pesados $2 \mathrm{~g}$ de biodiesel em um Erlenmeyer de $125 \mathrm{~mL}$ em balança analítica e se adicionou $25 \mathrm{ml}$ de éter etílico, medito por uma proveta, seguido de 2 gotas de fenolftaleína. A solução foi titulada com o auxílio de uma bureta digital com solução de hidróxido de sódio $0,1 \mathrm{~mol} / \mathrm{L}$ até o ponto de viragem, que é o aparecimento da coloração rósea. Anotou-se o valor de $\mathrm{NaOH}$ utilizado realizando triplicata.

As análises de enxofre, sódio, potássio, cálcio, magnésio e fósforo, foram realizados por meio de digestão ácida, com foi utilizado $1 \mathrm{~g}$ de biodiesel pesado em tubos de digestão de $25 \mathrm{~mm}$, foi adicionado $5 \mathrm{~mL}$ de ácido nítrico e levados para um bloco reator por 3 horas com a temperatura de $150^{\circ} \mathrm{C}$, após a digestão as amostras foram filtradas e diluídas com água purificada e analisadas no equipamento ICP-OES. 
A viscosidade foi obtida através de um viscosímetro capilar Cannon-Fenske em banho termostático a $40^{\circ} \mathrm{C}$, no qual consiste em medir o tempo necessário para que um volume de líquido flua pelo capilar sob a ação da gravidade e multiplicado este tempo pela constante do viscosímetro. 0 procedimento consistiu em, pré aquecimento e estabilização da temperatura a $40{ }^{\circ} \mathrm{C}$. Posteriormente colocou-se uma amostra de $40 \mathrm{~mL}$ de biodiesel no tubo viscosimétrico e em seguida fez-se a sucção da amostra, até o atingir o menisco, e inverteu-se o tubo para retirar o material em excesso. 0 tubo com a amostra foi imerso no banho termostatizado a $40{ }^{\circ} \mathrm{C}$ preso a uma garra num suporte de modo que o viscosímetro ficasse perfeitamente na vertical. Aproximadamente 15 minutos depois com a temperatura estabilizada, retirouse a pipeta para o líquido fluir. Quando o biodiesel alcançou no menisco de referência, iniciou-se a cronometragem do tempo até que foi atingido o outro ponto de referência. Esse tempo foi convertido em segundos e aplicado na seguinte fórmula:

$$
V=t \times k
$$

Onde: $V$ está para viscosidade $(\mathrm{mL} / \mathrm{s}), t$ está para tempo (segundos) e $k$ está para constante do viscosímetro a 40 o C $(0,036)$.

A análise do ponto de fulgor adotou o método de Cleveland, onde segue a norma ASTM D93. Para tal, $65 \mathrm{ml}$ do combustível é aquecido em recipiente aberto e sua taxa de aquecimento é verificada com a utilização de um termômetro. Uma chama se mantém posicionada próxima a superfície do fluido e o ponto de fulgor é dado pela temperatura em que ocorre a combustão dos gases desprendidos do biodiesel (Rodrigues et al., 2017).
Os dados foram submetidos à análise de variância (ANOVA) para as análises químicas e rendimento de biodiesel com significativa a $5 \%$ de probabilidade pelo teste Tukey. Nesta etapa utilizou-se o programa estatístico SISVAR versão 5.1 (Ferreira, 2003).

\section{Resultados e discussão}

A partir das análises realizada no biodiesel produzido, resultante da transesterificação dos óleos de soja cru e utilizado para fritura de alimento, obteve-se os resultados apresentados na Tabela 1. A densidade obtida para os óleos estudados ficaram em torno de $0,88 \mathrm{~g} / \mathrm{cm}^{3}$, sendo similar aos valores encontrados em literatura, $0,83 \mathrm{~g} / \mathrm{cm}^{3}$ (Lopes et al., 2011). Os resultados para densidade do biodiesel de soja, demonstraram resultados dentro da normatização da ANP.

A densidade do combustível deve ser conhecida, pois afeta diretamente o desempenho do motor e a emissão de gases, pois um combustível de alta densidade pode gerar fumaça negra e grande liberação de material particulado (Alptekin e Canakci, 2008).

O Ponto de fulgor foi determinado pela mínima temperatura que um líquido se torna inflamável, de acordo com Borsato et al. (2012), o biodiesel sem blenda de diesel comum ou seja biodiesel sem a adição de diesel derivado de petróleo (B100), apresentou um ponto de fulgor de $165^{\circ} \mathrm{C}$, portanto, apresenta pequena diferença em relação aos dados apresentados na Tabela 1 . 0 biodiesel apresenta a vantagem de reduzir a emissão de compostos de enxofre durante sua combustão, possui ponto de fulgor mais alto, o que lhe confere manuseio e armazenamento seguros e apresenta boa lubricidade (Knothe e Razon, 2017).

A viscosidade cinemática a $40{ }^{\circ} \mathrm{C}$ do biodiesel teve uma variação entre 4,0 
a $5,0 \mathrm{~mm}^{2} / \mathrm{s}$, estando em acordo com a referência da ANP como apresentado na Tabela 1. Simas (2008), analisando a viscosidade do biodiesel B100 a partir do óleo refinado de soja, apresentou 4,13 $\mathrm{mm}^{2} / \mathrm{s}$. Essa baixa viscosidade pode levar ao desgaste excessivo das partes auto lubrificantes do motor, uma diminuição no trabalho da bomba de combustível, entre outros problemas. Portanto, é muito importante que o biodiesel apresente uma viscosidade muito próxima do diesel.

A viscosidade cinemática é um parâmetro de elevada importância, que é utilizado como indicativo na conversão dos triglicerídeos em ésteres metílicos pela reação de transterificação, ocasionada pela quebra das cadeias maiores de triglicerídeos, diglicerideos e monoglicerideos a ésteres metílicos que possuem cadeias menores (Osawa et al., 2006).

Tabela 1. Resultados de biodiesel obtidos a partir do óleo de soja cru e de fritura.

\begin{tabular}{ccccc} 
& Unidade & Óleo Cru & Óleo Fritura & ANP \\
\hline Densidade & $\mathrm{g} / \mathrm{mL}$ & $0,871 \mathrm{a}$ & $0,883 \mathrm{a}$ & Não consta \\
Massa específica $20^{\circ} \mathrm{C}$ & $\mathrm{kg} / \mathrm{m}^{3}$ & $867 \mathrm{a}$ & $882 \mathrm{a}$ & $850-900$ \\
Índice de acidez & $\mathrm{mg} \mathrm{KOH} / \mathrm{g}$ & $0,09 \mathrm{a}$ & $0,12 \mathrm{a}$ & Max. 0,50 \\
Viscosidade Cinemática $40^{\circ} \mathrm{C}$ & $\mathrm{mm}^{2} / \mathrm{s}$ & $4,0 \mathrm{a}$ & $5,0 \mathrm{a}$ & $3,0-6,0$ \\
Ponto de fulgor & ${ }^{\circ} \mathrm{C}$ & $140 \mathrm{a}$ & $160 \mathrm{a}$ & Min. $100^{\circ} \mathrm{C}$ \\
Enxofre & $\mathrm{mg} / \mathrm{kg}$ & $18 \mathrm{a}$ & $19 \mathrm{a}$ & Max. 50 \\
Sódio + Potássio & $\mathrm{mg} / \mathrm{kg}$ & $1,9 \mathrm{a}$ & $2,2 \mathrm{a}$ & Max. 5,0 \\
Cálcio + Magnésio & $\mathrm{mg} / \mathrm{kg}$ & $1,2 \mathrm{a}$ & $1,8 \mathrm{a}$ & Max. 5,0 \\
Fósforo & $\mathrm{mg} / \mathrm{kg}$ & $0,89 \mathrm{a}$ & $1,01 \mathrm{a}$ & Max. 10,00
\end{tabular}

Letras iguais na linha, para a mesma característica, indicam que não teve diferenças estatisticamente significativas a $5 \%$ de probabilidade pelo teste de Tukey.

Os resultados acima mostram um nível baixíssimo de acidez em todas as amostras

analisadas e conforme a resolução

07 da ANP, considera-se um biodiesel padrão que apresente no máximo de 0,5 $\mathrm{mg} \mathrm{KOH/g.} \mathrm{Os} \mathrm{resultados} \mathrm{dos} \mathrm{íons}$ metálicos não excederam o valor máximo determinado pela ANP, os íons metálicos são introduzidos no biodiesel durante o processo de produção, contaminação, armazenamento ou podem vir originalmente da matéria-prima utilizadas na síntese. A acidez elevada pode catalisar reações intermoleculares dos triacilgliceróis, ao mesmo tempo em que afeta a estabilidade térmica do combustível na câmara de combustão. Também no caso do emprego carburante do óleo, a elevada acidez livre tem ação corrosiva sobre os componentes metálicos do motor (Marques et al., 2019).

Catalisadores alcalinos tais como hidróxido e metóxido de sódio e potássio são comumente utilizados na preparação comercial de biodiesel, de modo que os íons sódio e potássio podem estar presentes no biodiesel devido à purificação insuficiente do produto final. Estes íons podem estar presentes como 
sólidos abrasivos ou sabões solúveis, podendo contribuir para o desgaste das peças que compõem o motor (CHAVES et al., 2010). LIMA e FLUMIGNAN analisaram amostras comerciais de biodiesel e todas as amostras mostraram-se dentro das especificações exigidas pela ANP. 0 cálcio foi o elemento com maior concentração nas 23amostras analisadas, com valor de $1,0 \mathrm{mg} / \mathrm{kg}$, enquanto as concentrações de sódio e potássio foram em torno de 0,1 a 0,2 $\mathrm{mg} / \mathrm{kg}$.

No caso dos íons cálcio e magnésio, os mesmos podem ser introduzidos no biodiesel durante a purificação através da lavagem com água dura. A presença destes íons pode levar à formação de sabões insolúveis, dando origem a incrustações, o que pode prejudicar o funcionamento do motor.

Tabela 2. Resultados de rendimento de biodiesel obtidos a partir do óleo de soja cru e de fritura.

\begin{tabular}{ccc}
\hline Biodiesel & Óleo Cru & Óleo Fritura \\
\cline { 2 - 3 } Rendimento de biodiesel (\%) & $93,80 \mathrm{a}$ & $89,60 \mathrm{a}$ \\
\hline
\end{tabular}

Letras iguais na linha, para a mesma característica, indicam que não teve diferenças estatisticamente significativas a $5 \%$ de probabilidade pelo teste de Tukey.

Quanto ao rendimento do biodiesel os dois tipos de óleo se mostraram eficientes, o rendimento para o óleo cru foi maior devido não ter impurezas, o óleo de fritura devido ao seu uso no preparo de alimentos acaba por sua vez se tornando um óleo mais sujo com resíduo de alimentos e até mesmo um pouco de umidade, nesse quesito é interessante que o óleo vegetal tenha maior conversão possível em biodiesel já que é o produto de maior interesse econômico e o glicerol entra como um subproduto, apesar de suas inúmeras aplicações seu uso ainda é considerado incipiente e de baixo valor agregado (Battisti et al., 2017).

Ferrari et al. (2005), avaliando a taxa de conversão do óleo neutro de soja em éster etílico (biodiesel) e o rendimento no processo de transesterificação, encontraram valores muito próximos do respectivo trabalho algo em torno de $97,5 \%$ de biodiesel. Batista et al. (2015) usando óleo de soja e empregando catalisadores heterogêneos contendo potássio como $\mathrm{KOH}$ teve um rendimento de conversão em biodiesel de 96,5\%. Marques et al.
(2019), obteve um rendimento de $84,71 \%$ para o biodiesel proveniente do óleo de soja in natura e $81,39 \%$ no biodiesel proveniente do óleo de soja residual.

\section{Conclusões}

Resultados avaliados mostraram que o biodiesel produzido de ambas as fontes avaliadas está de acordo com normas regulamentadoras dispostas pela ANP. Tornando assim estes produtos tecnicamente viáveis para comercialização.

0 rendimento na produção do biodiesel oriundo de óleo refinado cru de soja foi $4,5 \%$ superior ao do óleo residual do processo de fritura de alimento, $93,80 \%$ e $89,60 \%$ respectivamente. Porem os dois tipos de óleo avaliados mostraram-se com uma ótima conversão em biodiesel.

\section{Referências}

ALPTEKIN, E.; CANAKCI, M. Determination of the density and the viscosities of biodiesel-diesel fuel blends. 
Renewable Energy, v. 33, n. 12, p. 26232630, 2008.

ANP - AGÊNCIA NACIONAL DO
PETRÓLEO, GÁS NATURAL E
BIOCOMBUSTÍVEIS. RESOLUÇÃO ANP
No 4, DE 2.2.2010 disponivel em :
<http://legislacao.anp.gov.br/?path=leg
islacao-anp/resol-
$\underline{\text { anp/2010/fevereiro\&item=ranp-4-- }}$
$\underline{\text { 2010> Acesso em: } 29 . \text { jul. } 19}$

ANP. Biodiesel. Disponível em: <http://www.anp.gov.br/biocombustiv eis/biodiesel>. Acesso em: 24 jul. 2019.

ANP. Vendas de combustíveis em 2018 ficam estáveis com relação a 2017. Disponível em: <http://www.anp.gov.br/noticias/5048 -vendas-de-combustiveis-em-2018ficam-estaveis-com-relacao-a-2017>. Acesso em: 24 jul. 2019.

BATISTA, P. S., RODRIGUES, G. O., RODRIGUES, B. G., SOUSA, M. N. Produção de Biodiesel a partir de óleo de soja empregando catalisadores heterogêneos contendo potássio. Blucher Chemical Engineering Proceedings, v. 1, n. 2, p. 10368-10375, 2015.

BATTISTI, G., JÚNIOR, E. S., DAL POZZO, D. M., SANTOS, R. F. Comparação das características físico-químicas do biodiesel de citronela e eucalipto com 0 biodiesel da soja. Acta Iguazu, v. 6, n. 5, p. 173-180, 2017.

BORSATO, D., MAIA, E. C. R., DALL'ANTONIA, L. H., SILVA, H. C. D., PEREIRA, J. L. Kinetics of oxidation of biodiesel from soybean oil mixed with TBHQ: determination of storage time. Química Nova, v. 35, n. 4, p. 733-737, 2012.

CHAVES, E. S., DOS SANTOS, E. J., ARAUJO, R. G., OLIVEIRA, J. V., FRESCURA, V. L. A., CURTIUS, A. J. Metals and phosphorus determination in vegetable seeds used in the production of biodiesel by ICP OES and ICP-MS. Microchemical Journal, v. 96, n. 1, p. 71-76, 2010.

DE SOUZA, Vinícius Heidemann; PACHECO, Sabrina Moro Villela; JÚNIOR, Américo Cruz. Viabilidade do uso de óleos vegetais usados em frituras para a produção de biodiesel. Revista Técnico Científica do IFSC, v. 2, n. 7, p. 27-34, 2019.

FERRARI, R. A.; OLIVEIRA, V. S.; SCABIO, A. Biodiesel de soja - taxa de conversão em ésteres etílicos, caracterização físico químico e consumo em gerador de energia. Revista Química Nova. São Paulo, v. 28, n. 1, p. 19-23, 2005.

FERREIRA, D. F. Sisvar: sistema de análise de variância para dados balanceados, versão 5.1. Lavras: UFLA/DEX, 2003.

JOSÉ SOLLA; FRANCISCO NÍLSON MOREIRA COSTA E; SILVA (ORGS.). Cadernos de sustentabilidade da Rio+20: diretrizes de sustentabilidade e guia de boas práticas da organização da Conferência das Nações Unidas sobre Desenvolvimento Sustentável.

Brasilia: [s.n.].

KNOTHE, G.; RAZON, L. F. Biodiesel fuels. Progress in Energy and Combustion Science, v. 58, p. 36-59, 2017.

LAM, M.K., TAN, K.T., LEE, K.T., MOHAMED, A.R. Malaysian palm oil: Surviving the food versus fuel dispute for a sustainable future. Renewable and Sustainable Energy Reviews, v. 13, n. 67, p. 1456-1464, 2009.

LIMA, L. P.; LOPES, A.; OLIVEIRA, M.C.J.; NEVES, M.C.T.; KOIKE, G.H.A. Comparativo entre biodiesel de dendê e tucumã no desempenho operacional de 
trator agrícola. Engenharia na Agricultura, Viçosa, MG, v. 20, n. 3, p. 234-243, 2012.

LIMA, V. S.; FLUMIGNAN, D. L. Desenvolvimento e avaliação do método analítico para determinação dos teores de sódio, potássio e cálcio em biodiesel por fotometria de chama. Revista Brasileira de Energias Renováveis, v. 6, n.1, p.21-28, 2017.

LOPES, A.; LIMA, L.P.; OLIVEIRA, M.C.G.; NEVES, M.C.T.; KOIKE, G.H.A. Densidade de biodiesel de girassol em função da temperatura e proporções de mistura density of biodiesel. Semana de Tecnologia do Curso de Biocombustíveis da Faculdade de Tecnologia de Jaboticabal. Suplemento. Ciência e Tecnologia: FATEC-JB, Jaboticabal, v.3, 2011.

LOPES, A.; SENÔ, M.A.A.F.; CAMARA, F.T.; FURLANI, C.E.A.; SILVA, R.P.; CORTEZ, J.W. Biodiesel no Brasil: aspectos gerais e síntese da legislação. Atualidades Jurídicas, v.1, p.1-19, 2008.

MARQUES, F. C.; ALVES, I. L.; JUNIOR, E. R.; DE OLIVEIRA, P. J. P.; REPOSSI, B. F. Produção de biodiesel utilizando óleo de soja descartado por estabelecimento comercial alocado no ifes, campus Cachoeiro de Itapemirim-ES. Revista Ifes Ciência, v. 5, n. 2, p. 253-267, 2019.

MARULANDA-BUITRAGO, Paola-Andrea; MARULANDA-CARDONA, VíctorFernando. Supercritical transesterification of beef tallow for biodiesel production in a batch reactor. CT\&F-Ciencia, Tecnología y Futuro, v. 6, n. 2, p. 57-68, 2015.

OLIVEIRA, L. R. DE et al. Sustentabilidade: da evolução dos conceitos à implementação como estratégia nas organizações. Niterói: ProDução, 2012.
OSAWA,C. C; GONCALVES, L. A. G.; RAGAZZI, S. Titulação potenciométrica aplicada na determinação de ácidos graxos livres de óleos e gorduras comestíveis. Quím. Nova. v.29, n.3, p. 593-599, 2006.

RODRIGUES, L. et al. Determinação do Ponto de Fulgor de Biodiesel Derivado de óleo de Palmiste (Elaeis Guineenses) e Macaúba (Acrocomia Aculeata) e Seus Derivadors. Poços de Caldas: [s.n.]. Disponível em: <http://quimicanova.sbq.org.br/imageb ank/pdf/Vol32No6_1596_43-

RV08446.pdf>. Acesso em: 24 jul. 2019.

SILVA, P. R. F.; FREITAS, T. F. S., Biodiesel: o ônus e o bônus de produzir combustível. Ciência Rural, v. 38 p. 843$851,2008$. 